QUARTERLY OF APPLIED MATHEMATICS

VOLUME LXV, NUMBER 3

SEPTEMBER 2007, PAGES 405-424

S 0033-569X(07)01045-9

Article electronically published on May 21, 2007

\title{
A CONTROL THEORETIC APPROACH TO THE SWIMMING OF MICROSCOPIC ORGANISMS
}

\author{
BY \\ JORGE SAN MARTÍN (Departamento de Ingeniería Matemática, Universidad de Chile, Casilla \\ 170/3 - Correo 3, Santiago, Chile), \\ TAKÉO TAKAHASHI (Institut Élie Cartan UMR7502, Université Henri Poincaré Nancy 1, BP239, \\ 54506 Vandouvre-lès-Nancy Cedex, France), \\ AND \\ MARIUS TUCSNAK (Institut Élie Cartan UMR7502, Université Henri Poincaré Nancy 1, BP239, \\ 54506 Vandoeuvre-lès-Nancy Cedex, France) \\ Abstract. In this paper, we give a control theoretic approach to the slow self- \\ propelled motion of a rigid body in a viscous fluid. The control of the system is the \\ relative velocity of the fluid with respect to the solid on the boundary of the rigid body \\ (the thrust). Our main results show that there exists a large class of finite-dimensional \\ input spaces for which the system is exactly controllable, i.e., one can find controls steer- \\ ing the rigid body into any final position with a prescribed velocity field. The equations \\ we use are motivated by models of swimming of micro-organisms like cilia. We give a \\ control theoretic interpretation of the swimming mechanism of these organisms, which \\ takes place at very low Reynolds numbers.
}

1. Introduction and main results. This paper is aimed at contributing to the understanding of the mechanism of swimming of some microscopic organisms from a control theoretic point of view. As already remarked in Taylor 20, for microscopic organisms the inertia forces, "which are the essential element in self-propulsion of all large living or mechanical bodies, are small compared with forces due to viscosity". The question of understanding the mechanism of swimming of microscopic organisms received considerable attention from both biologists and specialists in fluid mechanics (see, for instance, 20, Lighthill [13, Childress [5], Galdi 9] and the references therein).

An important example of swimming microscopic organisms is furnished by ciliata (see, for instance, Blake [2] or Brennen [3]). We recall, following Galdi [8], 9], that these

Received July 25, 2005.

2000 Mathematics Subject Classification. Primary 76D05.

E-mail address: jorge@dim.uchile.cl

E-mail address: takahash@iecn.u-nancy.fr

E-mail address: tucsnak@loria.fr

(C) 2007 Brown University Reverts to public domain 28 years from publication 
organisms can be seen as rigid bodies covered by a large number of hair-like organelles called cilia which move in a rather complicated way (see Blake and Otto [1] or Brennen and Winet [4]). In a commonly accepted model (the layer model), the rather complex motion of cilia is replaced by a velocity field on a surface enclosing the layer of cilia (see, for instance, Keller and $\mathrm{Wu}$ [1]).

In this work we propose a model of the motion of such micro-organisms consisting of a dynamical system whose state at instant $t$ is

$$
Z(t)=\left(\begin{array}{c}
\xi(t) \\
\omega(t) \\
\zeta(t) \\
R(t)
\end{array}\right),
$$

where $\xi(t)$ (respectively $\zeta(t)$ ) denotes the velocity (respectively the position) of the mass center of the rigid body and $\omega(t)$ (respectively $R(t)$ ) represents the angular velocity vector (respectively the rotation matrix with respect to a reference orientation) of the rigid body at instant $t$.

The system is controlled by the velocity field induced by the motion of cilia. From the mathematical point of view this control can be seen as the difference of the velocities of the fluid and of the solid on the boundary of the rigid body (the thrust). In order to be more precise, let us denote by $S(t) \subset \mathbb{R}^{3}$ the open bounded set representing the domain occupied by the moving organisms at instant $t$. The fact that the solid has a rigid motion implies that there exists an open bounded set $S \subset \mathbb{R}^{3}$ (which will be used as a reference configuration of the solid) such that

$$
S(t)=R(t) S+\zeta(t)
$$

for all $t \geq 0$, where $R(t)$ is an orthogonal matrix. We assume that the body is surrounded by a viscous incompressible fluid that occupies the domain $F(t)=\mathbb{R}^{3} \backslash \overline{S(t)}$, and we denote by $v(y, t)$ the velocity field of the fluid written in a coordinate attached to the rigid body $\left(y \in F=\mathbb{R}^{3} \backslash \bar{S}\right)$. The input function $u=\left(u_{1}, \ldots, u_{k}\right):[0, \infty) \rightarrow \mathbb{R}^{k}$ acts via the boundary condition on $\partial S$ by

$$
v(y, t)=\xi(t)+\omega(t) \times y+\sum_{i=1}^{k} u_{i}(t) \psi_{i}(y) \quad y \in \partial S, \quad t \geq 0 .
$$

The family of functions $\Psi=\left\{\psi_{1}, \ldots, \psi_{k}\right\}$ is supposed to be given and contained in one of the following spaces:

$$
\begin{gathered}
\mathcal{U}=\left\{\varphi \in C^{2}\left(\partial S ; \mathbb{R}^{3}\right) \mid \varphi=0 \text { outside } \Gamma\right\} \\
\mathcal{V}=\left\{\varphi \in C^{2}\left(\partial S ; \mathbb{R}^{3}\right) \mid \varphi \cdot n=0 \text { on } \partial S\right\} \\
\mathcal{W}=\mathcal{U} \cap \mathcal{V},
\end{gathered}
$$

where $\Gamma$ is an open subset of $\partial S$ and where, for $x \in \partial S, n(x)$ denotes the unit vector normal to $\partial S$ and oriented towards the interior of $\partial S$. This means that we are looking for input functions with values in a finite-dimensional vector space and possibly satisfying constraints (such as being tangential to $\partial S$ or being supported in a subset $\Gamma$ of $\partial S$ ). We endow $\mathcal{U}, \mathcal{V}$ and $\mathcal{W}$ with the usual $C^{2}$ topology so that they become Banach spaces. 
In the next section, we introduce a simplified model of a self-propelled body based on the above assumption (1.2) and on the balance laws of linear and angular momentum. In this model the state trajectory $Z$, defined in (1.1), satisfies a first-order differential system of the form

$$
\dot{Z}(t)=f(Z(t))+B_{\Psi} u(t), \quad t \geq 0 .
$$

Above $f: \mathbb{R}^{9} \times S O(3) \rightarrow \mathbb{R}^{9} \times M_{3}(\mathbb{R})$ is a smooth function (depending only on $S$ ), $B: \mathbb{R}^{k} \rightarrow \mathbb{R}^{9} \times M_{3}(\mathbb{R})$ is the input operator (depending on $S$ and on the choice of the family $\Psi$ ), and $S O(3)$ denotes the group of rotations in $\mathbb{R}^{3}$. The precise definitions of the functions $f$ and $B$ will be given in the next section. For $T \geq 0$, we recall that a system of the form (1.6) is said to be controllable in time $T$ if, for every $Z_{0}, Z_{1} \in \mathbb{R}^{9} \times S O(3)$ there exists an input function $u \in L^{2}\left(0, T ; \mathbb{R}^{k}\right)$ such that the solution $Z$ of (1.6) satisfies

$$
Z(0)=Z_{0}, \quad Z(T)=Z_{1}
$$

The first main result of this paper is the following.

Theorem 1.1. Assume that the boundary of $S$ is of class $C^{2}$, that $\Gamma$ is an arbitrary open subset of $\partial S$ and that $k=6$. Let $\mathcal{Y}_{1}$ be the subset of those $\Psi=\left(\psi_{1}, \ldots, \psi_{6}\right) \in \mathcal{U}^{6}$ such that the system (1.6) is controllable in any time $T>0$ and let $\mathcal{Y}_{2}$ be the subset of those $\Psi \in \mathcal{V}^{6}$ such that the system (1.6) is controllable in any time $T>0$. Then $\mathcal{Y}_{1}$ $\left(\right.$ respectively $\mathcal{Y}_{2}$ ) contains an open dense subset of $\mathcal{U}^{6}$ (respectively of $\mathcal{V}^{6}$ ).

The above result says that the motion of $S$ can be controlled for a "large" choice of $\Psi$ with velocity fields that are supported in $\Gamma$ or they are tangential to the boundary. The second main result shows that, by assuming that $\partial S$ is analytic we can control the motion of $S$ with velocity fields that are both tangential to the boundary and vanishing outside $\Gamma$.

TheOREm 1.2. Assume that the boundary of $S$ is analytic, that $\Gamma$ is an arbitrary open subset of $\partial S$ and that $k=6$. Let $\mathcal{Y}_{3}$ be the subset of those $\Psi \in \mathcal{W}^{6}$ such that the system (1.6) is controllable in any time $T>0$. Then $\mathcal{Y}_{3}$ contains an open dense subset of $\mathcal{W}^{6}$.

Moreover, we give several examples of families $\Psi$ that ensure the controllability property.

The plan of this paper is as follows. In Section 2 we describe the mathematical model. In Section 3 we show that our model reduces to a finite-dimensional dynamical system. Section 4 contains the proof of our main results. Finally, in Section 5 we give some examples of families $\Psi$ for which the system (1.6) is controllable.

2. The mathematical model. The full system modelling the motion of a rigid body into a viscous incompressible fluid is composed of the nonstationary Navier-Stokes equations for the fluid coupled to ordinary differential equations (coming from Newton's laws) 
for the rigid body. More precisely the system is described by the following equations:

$$
\begin{aligned}
\rho_{F}\left(\frac{\partial \bar{v}}{\partial t}+(\bar{v} \cdot \nabla) \bar{v}\right)-\mu \Delta \bar{v}+\nabla \bar{p}=0, & x \in F(t), t \in(0, T), \\
\operatorname{div} \bar{v}=0, & x \in F(t), t \in(0, T), \\
\bar{v}(x, t)=\bar{\xi}(t)+\bar{\omega}(t) \times(x-\zeta(t))+\bar{U}, & x \in \partial S(t), t \in[0, T], \\
\lim _{|y| \rightarrow \infty} \bar{v}(y)=0, & t \in(0, T), \\
m \dot{\bar{\xi}}(t)=-\int_{\partial S(t)} \sigma(\bar{v}, \bar{p}) n \mathrm{~d} \Gamma, & t \in(0, T), \\
\frac{\mathrm{d}}{\mathrm{d} t}(\bar{J} \bar{\omega})(t)=-\int_{\partial S(t)} y \times \sigma(\bar{v}, \bar{p}) n \mathrm{~d} \Gamma, & t \in(0, T), \\
\dot{\zeta}(t)=\bar{\xi}(t), & t \in(0, T), \\
\dot{R}(t)=\mathbb{S}(\bar{\omega}(t)) R(t) & t \in(0, T) .
\end{aligned}
$$

The domains $S(t)$ and $F(t)$ are defined by

$$
S(t)=\left\{x \in \mathbb{R}^{3}: x=R(t) y+\zeta(t), \quad y \in S\right\}, \quad F(t)=\mathbb{R}^{3} \backslash \bar{S}(t) .
$$

We can assume, without loss of generality, that the mass center of $S$ is located at the origin. In this case, the unknowns $\zeta(t) \in \mathbb{R}^{3}$, respectively $R(t) \in S O(3)$, in the above system stand for the position vector of the mass center, respectively the orientation matrix, of the solid $S(t)$. The other unknowns in the above system are the velocity field of the fluid $\bar{v}$, the pressure field in the fluid $\bar{p}$, the linear velocity of the mass center of the solid $\bar{\xi}$ and the angular velocity of the solid $\bar{\omega}$. Moreover, we have denoted by $\sigma(v, p)$ the stress tensor (also called the Cauchy stress), which is defined by

$$
\sigma(v, p)=-p I_{3}+2 \mu D(v),
$$

where $I_{3}$ is the identity matrix of $M_{3}(\mathbb{R})$ and $D(v)$ is the tensor field defined by

$$
D(v)_{k, l}=\frac{1}{2}\left(\frac{\partial v_{k}}{\partial y_{l}}+\frac{\partial v_{l}}{\partial y_{k}}\right) .
$$

The positive constant $\mu$ is the dynamical viscosity of the fluid. We have denoted by $\rho_{F}$ the positive density of the fluid. The constant $m$ is the mass of the rigid body whereas $\bar{J}$ denotes the inertia matrix of the rigid body. If we denote by $\rho>0$ the density of the solid, then we have that

$$
m=\int_{S} \rho \mathrm{d} x, \quad \bar{J}_{i, j}=\int_{S(t)} \rho\left(e_{i} \times(x-\bar{\zeta})\right) \cdot\left(e_{j} \times(x-\bar{\zeta})\right) \mathrm{d} x
$$

Moreover, for any function $w$ depending only on time, we have denoted by $\dot{w}$ its time derivative.

In (2.8) we have denoted

$$
\mathbb{S}(\omega)=\left(\begin{array}{ccc}
0 & \omega_{3} & -\omega_{2} \\
-\omega_{3} & 0 & \omega_{1} \\
\omega_{2} & -\omega_{1} & 0
\end{array}\right) \quad\left(\omega \in \mathbb{R}^{3}\right)
$$


It is well known that the application $\mathbb{S}$ is an isomorphism from $\mathbb{R}^{3}$ onto the space $A(3)$ of skew-symmetric matrices and that equation (2.8) could be equivalently written as

$$
\dot{R}(t) x=\bar{\omega}(t) \times(R(t) x) \quad\left(x \in \mathbb{R}^{3}\right) .
$$

Equations (2.1)-(2.8) determine an infinite-dimensional nonlinear system. Moreover, since the domain filled by the fluid is not a priori known, we have here a free boundary problem. Therefore the study of (2.1)-(2.8) is a difficult mathematical question. The wellposedness of this system has been extensively studied in recent literature (see, for instance, [15], [17, or [19]). However, questions such as controllability or stabilizability of (2.1)-(2.8) are (in the above infinite-dimensional setting) open questions. In order to tackle these questions, we derive a simplified finite-dimensional model (still nonlinear) aimed to approximate (2.1)-(2.8) in the case of slow motions (in a sense which will be made precise later).

Since the equations (2.1)-(2.8) are not written in a cylindrical domain, it is classical (see, for instance, Serre [16]) to use the following change of variables to transform the equations for the fluid into a system written in the fixed domain $F=\mathbb{R}^{3} \backslash \bar{S}$ :

$$
\begin{gathered}
y=R^{*}(t)(x-\zeta(t)), \\
v(y, t)=R^{*}(t) \bar{v}(\zeta(t)+R(t) y, t), \quad p(y, t)=\bar{p}(\zeta(t)+R(t) y, t), \\
\xi(t)=R^{*}(t) \bar{\xi}(t), \quad \omega(t)=R^{*}(t) \bar{\omega}(t) .
\end{gathered}
$$

The above functions satisfy the following problem:

$$
\begin{aligned}
\rho_{F}\left(\frac{\partial v}{\partial t}+([v-\xi-\omega \times y] \cdot \nabla) v+\omega \times v\right)-\mu \Delta v+\nabla p=0, & y \in F, t \in(0, T), \\
\operatorname{div} v=0, & y \in F, t \in(0, T), \\
v(y, t)=\xi(t)+\omega(t) \times y+U, & y \in \partial S, t \in[0, T], \\
\lim v(y)=0, & t \in(0, T), \\
m \dot{\xi}(t)=-\int_{\partial S} \sigma(v, p) n \mathrm{~d} \Gamma-m \omega \times \xi, & t \in(0, T), \\
J \dot{\omega}(t)=(J \omega) \times \omega-\int_{\partial S} y \times \sigma(v, p) n \mathrm{~d} \Gamma, & t \in(0, T), \\
\dot{\zeta}(t)=R(t) \xi(t), & t \in(0, T), \\
\dot{R}(t)=R(t) \mathbb{S}(\omega(t)) & t \in(0, T) .
\end{aligned}
$$

The above system can be written in dimensionless variables. More precisely, following [5], we consider some characteristic length $L$, some characteristic time $\tau$, and some characteristic speed $V$. We define the following dimensionless variables:

$$
\begin{gathered}
t^{*}=\tau^{-1} t, \quad y^{*}=L^{-1} y, \quad v^{*}=V^{-1} v, \quad p^{*}=L(V \mu)^{-1} p, \\
\xi^{*}=V^{-1} \xi, \quad \omega^{*}=L V^{-1} \omega, \quad \zeta^{*}=L^{-1} \zeta \\
m^{*}=L^{-3} m, \quad J^{*}=L^{-5} J, \quad T^{*}=\tau^{-1} T, \quad U^{*}=V^{-1} U .
\end{gathered}
$$


Then the above system can be written as

$$
\begin{gathered}
\operatorname{Re} \Sigma \frac{\partial v^{*}}{\partial t^{*}}+\operatorname{Re}\left(\left(\left[v^{*}-\xi^{*}-\omega^{*} \times y^{*}\right] \cdot \nabla^{*}\right) v^{*}+\omega^{*} \times v^{*}\right)-\Delta v^{*}+\nabla^{*} p^{*}=0, \\
y^{*} \in F^{*}, t^{*} \in\left(0, T^{*}\right), \\
\operatorname{div}^{*} v^{*}=0, \quad y^{*} \in F^{*}, t^{*} \in\left(0, T^{*}\right), \\
v^{*}\left(y^{*}, t^{*}\right)=\xi^{*}\left(t^{*}\right)+\omega^{*}\left(t^{*}\right) \times y^{*}+U^{*}, \quad y^{*} \in \partial S^{*}, t^{*} \in\left[0, T^{*}\right], \\
\lim _{\left|y^{*}\right| \rightarrow \infty} v^{*}\left(y^{*}\right)=0, \quad t^{*} \in\left(0, T^{*}\right), \\
\operatorname{Re} \Sigma \frac{m^{*}}{\rho_{F}} \dot{\xi^{*}}\left(t^{*}\right)=-\int_{\partial S^{*}} \sigma^{*}\left(v^{*}, p^{*}\right) n \mathrm{~d} \Gamma^{*}-\operatorname{Re} \Sigma \frac{m^{*}}{\rho_{F}} \omega^{*} \times \xi^{*}, \quad t^{*} \in\left(0, T^{*}\right), \\
\operatorname{Re} \Sigma \frac{J^{*}}{\rho_{F}} \dot{\omega}^{*}\left(t^{*}\right)=\operatorname{Re} \Sigma\left(\frac{J^{*}}{\rho_{F}} \omega^{*}\right) \times \omega^{*}-\int_{\partial S^{*}} y^{*} \times \sigma^{*}\left(v^{*}, p^{*}\right) n \mathrm{~d} \Gamma^{*}, \quad t^{*} \in\left(0, T^{*}\right), \\
\Sigma \dot{\zeta^{*}}\left(t^{*}\right)=R\left(t^{*}\right) \xi^{*}\left(t^{*}\right), \quad t^{*} \in\left(0, T^{*}\right), \\
\dot{R}\left(t^{*}\right)=R\left(t^{*}\right) \mathbb{S}\left(\omega^{*}\left(t^{*}\right)\right), \quad t^{*} \in\left(0, T^{*}\right) .
\end{gathered}
$$

In the above system, we have used the following dimensionless parameters:

$$
\begin{aligned}
\Sigma & =\frac{L}{\tau V} \quad \text { frequency parameter, } \\
\operatorname{Re} & =\frac{\rho_{F} V L}{\mu} \quad \text { Reynolds number. }
\end{aligned}
$$

In the case of the swimming of microscopic organisms, the above system can be simplified by using the fact that the motion of the fluid is a very slow one: the Reynolds number is, in this case, of the order of $10^{-1}$ and the frequency parameter of order 1 (see, for instance, Childress [5, ch.2]). Therefore, we neglect the first two terms in the right-hand side of (2.10) so that the motion of the fluid is modeled by the stationary linear Stokes equations. This means that, although the flow field is time dependent, insofar as the dynamics of the fluid is concerned, it is moving slowly (quasi-steady).

Concerning the solid part, depending on the relative magnitude of the density of the microscopic organisms (with respect to the density of the fluid) the term corresponding to the time derivative and the nonlinear terms in equations (2.14) and (2.15) could be neglected or not. In this paper, we do not make any assumption on the density $\rho$ of the solid and so we will keep all these terms. The mathematical analysis of the models obtained by neglecting these terms is quite similar to the analysis in the next sections so that most of our results would also apply for these models.

For the sake of simplicity, in the remaining part of the paper we omit the exponent $*$, we assume that $\Sigma=1$ and we use the notation $m$ and $J$ for the parameters

$$
m=\operatorname{Re} \frac{m^{*}}{\rho_{F}}, \quad J=\operatorname{Re} \frac{J^{*}}{\rho_{F}} .
$$


With the above assumptions the system (2.10)-(2.17) simplifies to

$$
\begin{aligned}
-\Delta v+\nabla p=0, & \text { in } F \times(0, T), \\
\operatorname{div} v=0, & \text { in } F \times(0, T), \\
v(y, t)=\xi(t)+\omega(t) \times y+\sum_{i=1}^{k} u_{i}(t) \psi_{i}(y), & y \in \partial S, t \in(0, T), \\
\lim _{|y| \rightarrow \infty} v(y)=0, & t \in(0, T), \\
m \dot{\xi}(t)=-\int_{\partial S} \sigma(v, p) n \mathrm{~d} \Gamma+m \omega \times \xi, & t \in(0, T), \\
J \dot{\omega}(t)=-\int_{\partial S} y \times \sigma(v, p) n \mathrm{~d} \Gamma+(J \omega) \times \omega, & t \in(0, T), \\
\dot{\zeta}(t)=R(t) \xi(t), & t \in(0, T), \\
\dot{R}(t)=R(t) \mathbb{S}(\omega(t)), & t \in(0, T), \\
\xi(0)=\xi_{0}, \omega(0)=\omega_{0}, & y \in F, \\
\zeta(0)=\zeta_{0}, \quad R(0)=R_{0}, & y \in F .
\end{aligned}
$$

The function $u=\left(u_{1}, \ldots, u_{k}\right) \in L^{2}\left(0, T ; \mathbb{R}^{k}\right)$ is the control of the system and $\Psi=$ $\left\{\psi_{1}, \ldots, \psi_{k}\right\}$ is a fixed subset of $C^{2}\left(\partial S ; \mathbb{R}^{3}\right)$. As shown in Section 3 equations (2.18)(2.27) determine a nonlinear finite-dimensional system. In the remaining part of this paper we study the controllability of this system and we no longer consider the infinitedimensional system (2.1)-(2.8).

3. Dynamical system formulation. The simplifying assumption of neglecting the term containing the derivative with respect to time in the fluid equation enables us to write (2.18)-(2.27) as a dynamical system in $\mathbb{R}^{9} \times S O(3)$. In order to make this assertion precise, we introduce some auxiliary fields (see [10, ch.5] or [8]). Assume that $\left(e_{i}\right)$ is an orthonormal basis of $\mathbb{R}^{3}$. We define $\left(h^{(i)}, p^{(i)}\right)$ (respectively $\left(H^{(i)}, P^{(i)}\right)$ ) as the solution of the following boundary value problem for the Stokes system:

$$
\left\{\begin{aligned}
-\Delta h^{(i)}+\nabla p^{(i)}=0, & \text { in } F, \\
\operatorname{div} h^{(i)}=0, & \text { in } F, \\
h^{(i)}(y)=e_{i}, & y \in \partial S, \\
\lim _{|y| \rightarrow \infty} h^{(i)}(y)=0, &
\end{aligned}\right.
$$

respectively

We denote

$$
\left\{\begin{aligned}
-\Delta H^{(i)}+\nabla P^{(i)}=0, & \text { in } F, \\
\operatorname{div} H^{(i)}=0, & \text { in } F, \\
H^{(i)}(y)=e_{i} \times y, & y \in \partial S, \\
\lim _{|y| \rightarrow \infty} H^{(i)}(y)=0 . &
\end{aligned}\right.
$$

$$
\begin{gathered}
g^{(i)}=\left.\sigma\left(h^{(i)}, p^{(i)}\right) n\right|_{\partial F}, \quad i \in\{1,2,3\}, \\
G^{(i)}=\left.\sigma\left(H^{(i)}, P^{(i)}\right) n\right|_{\partial F}, \quad i \in\{1,2,3\} .
\end{gathered}
$$


For homogeneous Sobolev spaces we use, following [7], the notation

$$
D^{l, q}(F)=\left\{u \in L_{\text {loc }}^{1}(F) \quad \mid \quad \partial^{\alpha} u \in L^{q}(F) \text { for all } \alpha \in \mathbb{N}^{3},|\alpha|=l\right\},
$$

with $l \in \mathbb{N}, 1 \leq q \leq \infty$.

The following result shows that the above systems are well posed.

Lemma 3.1. (1) Assume that the boundary $\partial S$ is of class $C^{2}$. Then the systems (3.1) and (3.2) admit unique solutions such that

$$
\begin{gathered}
h^{(i)}, \quad H^{(i)} \in L^{s}(F) \cap D^{1, r}(F) \cap D^{2, q}(F) \cap C^{\infty}(F), \\
p^{(i)}, P^{(i)} \in L^{r}(F) \cap D^{1, q}(F) \cap C^{\infty}(F)
\end{gathered}
$$

for $s \in(3, \infty], r \in\left(\frac{3}{2}, \infty\right], q \in(1, \infty)$. Moreover, we have that

$$
\begin{gathered}
\left\|(1+|y|) h^{(i)}\right\|_{L^{\infty}(F)}<\infty, \\
\left\|(1+|y|) H^{(i)}\right\|_{L^{\infty}(F)}<\infty .
\end{gathered}
$$

(2) Assume that the boundary $\partial S$ is analytic. Then $\left(h^{(i)}, p^{(i)}\right)$ and $\left(H^{(i)}, P^{(i)}\right)$ are analytic up to the boundary.

Proof. The first result comes from the classical wellposedness results for the Stokes system (see, for instance, [7, Chapter V], 17]). For the analyticity we refer to Komatsu [12] and Morrey [14.

We next introduce several matrices playing an important role in the remaining part of the paper. For $i, j \in\{1,2,3\}$ we denote

$$
\begin{aligned}
K_{i, j} & =-\int_{\partial S} g_{j}^{(i)} \mathrm{d} \Gamma, & C_{i, j} & =-\int_{\partial S}\left(x \times g^{(i)}\right)_{j} \mathrm{~d} \Gamma, \\
\widetilde{C}_{i, j} & =-\int_{\partial S} G_{j}^{(i)} \mathrm{d} \Gamma, & \Omega_{i, j} & =-\int_{\partial S}\left(x \times G^{(i)}\right)_{j} \mathrm{~d} \Gamma .
\end{aligned}
$$

It is known (see, for instance, [10, ch.5]) that $\widetilde{C}=C^{*}$ and that the matrix $A \in M_{6}(\mathbb{R}$ ) defined by

$$
A=\left(\begin{array}{cc}
m^{-1} K & m^{-1} C \\
J^{-1} C^{*} & J^{-1} \Omega
\end{array}\right)
$$

is selfadjoint and negative-definite, provided that we endow $\mathbb{R}^{6}$ with the inner product

$$
\langle a, b\rangle=m \sum_{q=1}^{3} a_{q} b_{q}+\sum_{p, q=1}^{3} J_{p, q} a_{q+3} b_{p+3} .
$$

We next introduce the matrices $B^{(1)}, B^{(2)} \in M_{3 \times k}(\mathbb{R})$ and $B \in M_{6 \times k}$ defined by

$$
\begin{gathered}
B_{i, j}^{(1)}=-\int_{\partial S} g^{(i)} \cdot \psi_{j} \mathrm{~d} \Gamma, \quad i \in\{1,2,3\}, \quad j \in\{1, \ldots, k\}, \\
B_{i, j}^{(2)}=-\int_{\partial S} G^{(i)} \cdot \psi_{j} \mathrm{~d} \Gamma, \quad i \in\{1,2,3\}, \quad j \in\{1, \ldots, k\}, \\
B=\left(\begin{array}{c}
m^{-1} B^{(1)} \\
J^{-1} B^{(2)}
\end{array}\right) .
\end{gathered}
$$


For given input functions $u_{1}, \ldots, u_{k} \in L^{2}(0, T ; \mathbb{R})$, according to [7, Chapter V], there exists a unique solution $(U, Q)$ of

$$
\begin{array}{rc}
-\Delta U+\nabla Q=0, & \text { in } F \times(0, T), \\
\operatorname{div} U=0, & \text { in } F \times(0, T), \\
U(y, t)=\sum_{i=1}^{k} u_{i}(t) \psi_{i}(y), & y \in \partial S, t \in(0, T), \\
\lim _{|y| \rightarrow \infty} U(y)=0, & t \in(0, T)
\end{array}
$$

satisfying

$$
\begin{gathered}
U(\cdot, t) \in L^{s}(F) \cap D^{1, r}(F) \cap D^{2, q}(F) \cap C^{\infty}(F), \\
Q(\cdot, t) \in L^{r}(F) \cap D^{1, q}(F) \cap C^{\infty}(F)
\end{gathered}
$$

for $s \in(3, \infty], r \in\left(\frac{3}{2}, \infty\right], q \in(1, \infty)$ and

$$
\underset{y \in F}{\operatorname{ess} \sup }(1+|y|)|U(y, t)|<\infty
$$

for almost every $t \in(0, T)$.

For $a, b \in \mathbb{R}^{3}$, we also set

$$
E\left(\left(\begin{array}{l}
a \\
b
\end{array}\right)\right)=\left(\begin{array}{c}
b \times a \\
J^{-1}((J b) \times b)
\end{array}\right) .
$$

We are now in a position to prove that equations (2.18)-(2.27) determine a finitedimensional dynamical system.

Lemma 3.2. Assume that $T>0$ and that $u \in L^{2}\left(0, T ; \mathbb{R}^{m}\right)$. Then $(v, p, \xi, \omega, \zeta, R)$ satisfy (2.18)-(2.27) together with

$$
\begin{gathered}
v \in H^{1}\left(0, T ; L^{s}(F) \cap D^{1, r}(F) \cap D^{2, q}(F) \cap C^{\infty}(F)\right), \\
p \in H^{1}\left(0, T ; L^{r}(F) \cap D^{1, q}(F) \cap C^{\infty}(F)\right), \\
\xi \in H^{1}\left(0, T ; \mathbb{R}^{3}\right), \omega \in H^{1}\left(0, T ; \mathbb{R}^{3}\right), \zeta \in C^{1}\left([0, T] ; \mathbb{R}^{3}\right), \quad R \in C^{1}([0, T] ; S O(3)),
\end{gathered}
$$

for $s \in(3, \infty], r \in\left(\frac{3}{2}, \infty\right], t \in(1, \infty)$ if and only if

$$
\begin{gathered}
\left(\begin{array}{c}
\dot{\xi}(t) \\
\dot{\omega}(t)
\end{array}\right)=A\left(\begin{array}{c}
\xi(t) \\
\omega(t)
\end{array}\right)+E\left(\left(\begin{array}{c}
\xi(t) \\
\omega(t)
\end{array}\right)\right)+B u(t), \\
\dot{\zeta}(t)=R(t) \xi(t), \quad \dot{R}(t)=R(t) \mathbb{S}(\omega(t)), \\
\xi(0)=\xi_{0}, \omega(0)=\omega_{0}, \zeta(0)=\zeta_{0}, R(0)=R_{0}, \\
v=\sum_{i} \xi_{i} h^{(i)}+\omega_{i} H^{(i)}+U, \quad p=\sum_{i} \xi_{i} p^{(i)}+\omega_{i} P^{(i)}+Q,
\end{gathered}
$$

where $A, B$ are the matrices defined by (3.7), (3.10), $E(\cdot)$ is defined by (3.15) and $(U, Q)$ is the solution of (3.11)-(3.14). 
Proof. Assume that $(v, p, \xi, \omega, \zeta, R)$ satisfies (3.19)-(3.22). From (3.1), (3.2) and (3.11)(3.14), it is easy to check that $(v, p)$ satisfies (2.18)-(2.20). From (3.19) and from the definitions of $A$ and $B$, it follows that for $k \in\{1,2,3\}$ we have

$$
\begin{gathered}
m \dot{\xi}_{k}=-\int_{\partial F} \sum_{i=1}^{3}\left(\xi_{i} g_{k}^{(i)}+\omega_{i} G_{k}^{(i)}\right) \mathrm{d} \Gamma-\int_{\partial S} \sum_{j=1}^{3} u_{j} \psi_{j} \cdot g^{(k)} \mathrm{d} \Gamma+m(\omega \times \xi)_{k}, \\
(J \dot{\omega})_{k}=-\int_{\partial F} \sum_{i=1}^{3}\left[\xi_{i}\left(y \times g^{(i)}\right)_{k}+\omega_{i}\left(y \times G^{(i)}\right)_{k}\right] \mathrm{d} \Gamma-\int_{\partial S} \sum_{j=1}^{3} u_{j} \psi_{j} \cdot G^{(k)} \mathrm{d} \Gamma+[(J \omega) \times \omega]_{k} .
\end{gathered}
$$

By using (3.3) and (3.13), the last term in the right-hand side of (3.23) can be written as

$$
\int_{\partial S} \sum_{j=1}^{3} u_{j} \psi_{j} \cdot g^{(k)} \mathrm{d} \Gamma=\int_{\partial S} \sigma\left(h^{(k)}, p^{(k)}\right) n \cdot U \mathrm{~d} \Gamma .
$$

The above relation, combined to (3.30) (proved in Lemma 3.4), implies that

$$
\int_{\partial S} \sum_{j=1}^{3} u_{j} \psi_{j} \cdot g^{(k)} \mathrm{d} \Gamma=\left[\int_{\partial S} \sigma(U, Q) n \mathrm{~d} \Gamma\right]_{k} .
$$

Relations (3.23) and (3.25) clearly imply that

$$
m \dot{\xi}=-\int_{\partial F} \sum_{i=1}^{3}\left(\xi_{i} g^{(i)}+\omega_{i} G^{(i)}\right) \mathrm{d} \Gamma-\int_{\partial F} \sigma(U, Q) n \mathrm{~d} \Gamma+m \omega \times \xi .
$$

Similar calculations show that

$$
J \dot{\omega}=-\int_{\partial F} \sum_{i=1}^{3}\left[\xi_{i}\left(y \times g^{(i)}\right)+\omega_{i}\left(y \times G^{(i)}\right)\right] \mathrm{d} \Gamma-\int_{\partial S} y \times \sigma(U, Q) n \mathrm{~d} \Gamma+(J \omega) \times \omega .
$$

Relations (3.26) and (3.27) imply that $(\xi, \omega)$ satisfies (2.22)-(2.23). Then, by taking into consideration the fact that $(\zeta, R)$ satisfy (2.24)-(2.27), we obtain that $(v, p, \xi, \omega, \zeta, R)$ satisfies (2.18) $-(2.27)$.

The proof of the fact that any solution of (2.18)-(2.27) satisfies (3.19)-(3.22) is similar and therefore we don't give it here.

From the above lemma it easily follows that the following result holds.

Corollary 3.3. Assume that $T>0$ and that $u \in L^{2}\left(0, T ; \mathbb{R}^{k}\right)$. Then there exists a unique solution $(v, p, \xi, \omega, \zeta, R)$ of the system (2.18)-(2.27) satisfying (3.16)-(3.18). More precisely, if we denote $z(t)=\left(\begin{array}{c}\xi(t) \\ \omega(t)\end{array}\right)$, then equations (2.18)-(2.27) determine a dynamical system with input space $\mathbb{R}^{k}$, state space $\mathbb{R}^{9} \times S O(3)$ and with the state equations

$$
\begin{gathered}
\dot{z}(t)=A z(t)+E(z(t))+B u(t), \\
\dot{\zeta}(t)=R(t) \xi(t), \quad \dot{R}(t)=R(t) \mathbb{S}(\omega(t)) .
\end{gathered}
$$


Proof. The local-in-time existence follows from the Cauchy-Lipschitz Theorem. To get the global-in-time existence it suffices to show that the solutions do not blow up in finite time. It is easy to check that $\langle E(z), z\rangle=0$ for every $z \in \mathbb{R}^{6}$. Therefore, by taking the inner product of (3.28) with $z(t)$ we have that

$$
\frac{1}{2} \frac{d}{d t}\|z(t)\|^{2}=\langle A z(t), z(t)\rangle+\langle B u(t), z(t)\rangle .
$$

The above relation and the fact that $A$ is symmetric and negative-definite imply that

$$
\|z(t)\|^{2} \leq\|z(0)\|^{2}+\int_{0}^{t}\left(\|B u(s)\|^{2}+\|z(s)\|^{2}\right) \mathrm{d} s .
$$

By the Gronwall lemma it follows that $z$ does not blow up in finite time so that we have proved the global existence result.

We have used in the proof of Lemma 3.2 the following technical lemma.

LEmma 3.4. With the above notation, for $i \in\{1,2,3\}$ and for almost every $t \in(0, T)$, we have that

$$
\begin{gathered}
\int_{\partial S} \sigma\left(h^{(i)}, p^{(i)}\right) n \cdot U \mathrm{~d} \Gamma=\left[\int_{\partial S} \sigma(U, Q) n \mathrm{~d} \Gamma\right]_{i}, \\
\int_{\partial S} \sigma\left(H^{(i)}, P^{(i)}\right) n \cdot U \mathrm{~d} \Gamma=\left[\int_{\partial S} x \times \sigma(U, Q) n \mathrm{~d} \Gamma\right]_{i} .
\end{gathered}
$$

Proof. By taking the inner product of (3.11) with $h^{(i)}$ we obtain that

$$
\int_{F} \operatorname{div} \sigma(U, Q) \cdot h^{(i)} \mathrm{d} y=0 .
$$

Using an integration by parts, the above relation implies that

$$
\int_{\partial S} \sigma(U, Q) n \cdot h^{(i)} \mathrm{d} \Gamma=\int_{F} D(U): D\left(h^{(i)}\right) \mathrm{d} y .
$$

Similarly, by taking the inner product of (3.1) with $U$ we obtain that

$$
\int_{F} \operatorname{div} \sigma\left(h^{(i)}, p^{(i)}\right) \cdot U \mathrm{~d} y=0
$$

Integrating by parts we get that

$$
\int_{\partial S} \sigma\left(h^{(i)}, p^{(i)}\right) n \cdot U \mathrm{~d} \Gamma=\int_{F} D(U): D\left(h^{(i)}\right) \mathrm{d} y .
$$

From (3.32) and 3.33) and the fact that $h^{(i)}=e_{i}$ on $\partial S$, we conclude that (3.30) holds true.

The proof of (3.31) is similar, so we skip it here. 
4. Proof of the main results. The main ingredients of the proof of Theorem 1.1 are the following two lemmas.

Lemma 4.1. Assume that $\Gamma$ is a nonempty open subset of $\partial S$ (with respect to the induced topology).

(1) Suppose that $\partial S$ is of class $C^{2}$. Then the family

$$
\mathcal{F}_{1}=\left\{g^{(1)}, g^{(2)}, g^{(3)}, G^{(1)}, G^{(2)}, G^{(3)}\right\}
$$

is linearly independent in $L^{2}\left(\Gamma, \mathbb{R}^{3}\right)$ and the family

$$
\mathcal{F}_{2}=\left\{\left(g^{(i)} \times n\right) \times n,\left(G^{(i)} \times n\right) \times n \mid i \in\{1,2,3\}\right\}
$$

is linearly independent in $L^{2}\left(\partial S, \mathbb{R}^{3}\right)$.

(2) Suppose that $\partial S$ is analytic. Then $\mathcal{F}_{2}$ is linearly independent in $L^{2}\left(\Gamma, \mathbb{R}^{3}\right)$.

Proof. (1) Let us consider $\gamma, \delta \in \mathbb{R}^{3}$ such that

$$
\sum_{1 \leq i \leq 3}\left(\gamma_{i} g^{(i)}+\delta_{i} G^{(i)}\right)=0 \quad \text { on } \Gamma .
$$

Then, we denote

$$
H=\sum_{1 \leq i \leq 3}\left(\gamma_{i} h^{(i)}+\delta_{i} H^{(i)}\right), \quad P=\sum_{1 \leq i \leq 3}\left(\gamma_{i} p^{(i)}+\delta_{i} P^{(i)}\right) .
$$

From (4.1) it follows that

$$
\sigma(H, P) n=0 \quad \text { on } \Gamma \text {. }
$$

Moreover, from the definitions (3.1) and (3.2) of $\left(h^{(i)}, p^{(i)}\right)$ and $\left(H^{(i)}, P^{(i)}\right)$ we have that $(H, P)$ satisfies

$$
\left\{\begin{aligned}
-\Delta H+\nabla P=0, & \text { in } F, \\
\operatorname{div} H=0, & \text { in } F, \\
H=\gamma+\delta \times y, & y \in \partial S, \\
\lim _{|y| \rightarrow \infty} H(y)=0 . &
\end{aligned}\right.
$$

We consider $\mathcal{O} \subset S$ to be an open set such that

$$
\overline{\mathcal{O}} \cap \bar{F} \subset \Gamma .
$$

We then define $\widetilde{H}$ and $\widetilde{P}$ on $F \cup \mathcal{O}$ by the following formulas: $\widetilde{H}=H-(\gamma+\delta \times y)$ for all $y \in F$ and $\widetilde{H}=0$ for all $y \in \mathcal{O}$ and $\widetilde{P}=P$ for all $y \in F$ and $\widetilde{P}=0$ for all $y \in \mathcal{O}$. By using (4.3) it follows that $(\widetilde{H}, P) \in H_{\mathrm{loc}}^{1}(F \cup \mathcal{O}) \times L_{\text {loc }}^{2}(F \cup \mathcal{O})$ and

$$
\begin{array}{rlrl}
-\Delta \widetilde{H}+\nabla \widetilde{P} & =0, & & \text { in } F \cup \mathcal{O}, \\
\operatorname{div} \widetilde{H} & =0, & \text { in } F \cup \mathcal{O}, \\
\widetilde{H} & =0, & \text { in } \mathcal{O} .
\end{array}
$$


By using the unique continuation result of Fabre and Lebeau [6, Theorem 1], we deduce that $\widetilde{H}=0$, so that $H=\gamma+\delta \times y$ for all $y \in F$. Since $\lim _{|y| \rightarrow \infty} H(y)=0$, we have that $\gamma=\delta=0$. Consequently, the family $\mathcal{F}_{1}$ is linearly independent.

For the linear independence of $\mathcal{F}_{2}$ in $L^{2}\left(\partial S, \mathbb{R}^{3}\right)$ we refer to Lemma 2.1 of 8 .

(2) Let us consider $\gamma, \delta \in \mathbb{R}^{3}$ such that

$$
\sum_{1 \leq i \leq 3}\left(\gamma_{i}\left(g^{(i)} \times n\right) \times n+\delta_{i}\left(G^{(i)} \times n\right) \times n\right)=0 \quad \text { on } \Gamma .
$$

Then, since the function

$$
x \mapsto \sum_{i=1}^{3}\left[\gamma_{i}\left(g^{(i)} \times n\right) \times n+\delta_{i}\left(G^{(i)} \times n\right) \times n\right]
$$

is analytic on $\partial S$, it follows that

$$
\sum_{i=1}^{3}\left[\gamma_{i}\left(g^{(i)} \times n\right) \times n+\delta_{i}\left(G^{(i)} \times n\right) \times n\right]=0, \quad \text { on } \partial S .
$$

The linear independence of $\mathcal{F}_{2}$ in $L^{2}\left(\Gamma, \mathbb{R}^{3}\right)$ follows now from the fact that $\mathcal{F}_{2}$ is linearly independent in $L^{2}\left(\partial S, \mathbb{R}^{3}\right)$.

The result below shows that for $k=6$ the mapping associating the matrix $B$ to the family $\left\{\psi_{1}, \ldots \psi_{6}\right\}$ via formulas (3.8)-(3.10) is, under quite general assumptions, onto.

Lemma 4.2. Assume that $\Gamma$ is a nonempty open subset of $\partial S$ (with respect to the induced topology). Then the linear operator $\Lambda:\left[L^{2}\left(\partial S ; \mathbb{R}^{3}\right)\right]^{6} \rightarrow M_{6 \times 6}(\mathbb{R})$, defined by

$$
\Lambda\left(\begin{array}{c}
\psi_{1} \\
\vdots \\
\psi_{6}
\end{array}\right)=-\left(\begin{array}{l}
\left(\int_{\partial S} g^{(i)} \cdot \psi_{j} \mathrm{~d} \Gamma\right)_{i \in\{1,2,3\}, j \in\{1, \ldots, 6\}} \\
\left(\int_{\partial S} G^{(i)} \cdot \psi_{j} \mathrm{~d} \Gamma\right)_{i \in\{1,2,3\}, j \in\{1, \ldots, 6\}}
\end{array}\right),
$$

is such that

(1) $\Lambda\left(\mathcal{U}^{6}\right)=\Lambda\left(\mathcal{V}^{6}\right)=M_{6 \times 6}(\mathbb{R})$;

(2) if $\partial S$ is analytic, then $\Lambda\left(\mathcal{W}^{6}\right)=M_{6 \times 6}(\mathbb{R})$.

Proof. We first set

$$
\begin{aligned}
& \mathbb{U}=\left\{\left(\varphi_{i}\right) \in L^{2}\left(\partial S ; \mathbb{R}^{3}\right)^{6} \mid \varphi_{i}=0 \text { outside } \Gamma, i \in\{1, \ldots, 6\}\right\}, \\
& \mathbb{V}=\left\{\left(\varphi_{i}\right) \in L^{2}\left(\partial S ; \mathbb{R}^{3}\right)^{6} \mid \varphi_{i} \cdot n=0 \text { on } \partial S, i \in\{1, \ldots, 6\}\right\}, \\
& \mathbb{W}=\mathbb{U} \cap \mathbb{V},
\end{aligned}
$$


and we denote by $\Lambda_{\mathbb{U}}$ (respectively $\Lambda_{\mathbb{V}}, \Lambda_{\mathbb{W}}$ ) the restriction of $\Lambda$ to $\mathbb{U}$ (respectively $\mathbb{V}$, $\mathbb{W})$. Simple calculations show that for all $C \in M_{6}(\mathbb{R})$ we have

$$
\begin{aligned}
& \Lambda_{\mathbb{U}}^{*}(C)=\left(\begin{array}{c}
\sum_{k=1}^{3}\left[C_{k, 1} g^{(k)}+C_{k+3,1} G^{(k)}\right] \\
\vdots \\
\sum_{k=1}^{3}\left[C_{k, 6} g^{(k)}+C_{k+3,6} G^{(k)}\right]
\end{array}\right) \text { on } \Gamma \\
& \Lambda_{\mathbb{V}}^{*}(C)=\left(\begin{array}{c}
\sum_{k=1}^{3}\left[C_{k, 1}\left(g^{(k)} \times n\right) \times n+C_{k+3,1}\left(G^{(k)} \times n\right) \times n\right] \\
\vdots \\
\sum_{k=1}^{3}\left[C_{k, 6}\left(g^{(k)} \times n\right) \times n+C_{k+3,6}\left(G^{(k)} \times n\right) \times n\right]
\end{array}\right) \quad \text { on } \partial S \\
& \Lambda_{\mathbb{W}}^{*}(C)=\left(\begin{array}{c}
\sum_{k=1}^{3}\left[C_{k, 1}\left(g^{(k)} \times n\right) \times n+C_{k+3,1}\left(G^{(k)} \times n\right) \times n\right] \\
\vdots \\
\sum_{k=1}^{3}\left[C_{k, 6}\left(g^{(k)} \times n\right) \times n+C_{k+3,6}\left(G^{(k)} \times n\right) \times n\right]
\end{array}\right) \text { on } \Gamma \text {. }
\end{aligned}
$$

We first prove that the restriction of $\Lambda$ to $\mathcal{U}^{6}$ is onto. By using Lemma 4.1 it follows that $\Lambda_{\mathbb{U}}^{*}$ is one to one. Consequently, the range of $\Lambda_{\mathbb{U}}$ is a dense subspace of $M_{6}(\mathbb{R})$. By using the density of $\mathcal{U}^{6}$ in $\mathbb{U}$ we get that $\Lambda\left(\mathcal{U}^{6}\right)$ is a dense subspace of $M_{6}(\mathbb{R})$. Since $M_{6}(\mathbb{R})$ is finite dimensional, all its subspaces are closed so that $\Lambda\left(\mathcal{U}^{6}\right)=M_{6}(\mathbb{R})$.

Using again Lemma 4.1, we have that $\Lambda_{\mathbb{V}}^{*}$ is one to one and if $\partial S$ is analytic we have that $\Lambda_{\mathbb{W}}^{*}$ is one to one. Acting as for $\mathcal{U}$, we easily conclude that $\Lambda\left(\mathcal{V}^{6}\right)=M_{6}(\mathbb{R})$ and that for $\partial S$ analytic we have that $\Lambda\left(\mathcal{W}^{6}\right)=M_{6}(\mathbb{R})$.

We are now in a position to prove the main results.

Proof of Theorem 1.1. We only prove Theorem 1.1 for the set $\mathcal{Y}_{1}$. The proof for the set $\mathcal{Y}_{2}$ is completely similar and it is omitted.

Let us define $\widetilde{\mathcal{Y}}_{1}$ to be the set of those $\Psi$ for which the matrix $B$ given by (3.8)-(3.9) is invertible. We first check that $\mathcal{Y}_{1}$ contains $\widetilde{\mathcal{Y}}_{1}$ and then that $\widetilde{\mathcal{Y}}_{1}$ is an open dense subset of $\mathcal{U}^{6}$. Indeed, assume that

$$
Z_{0}=\left(\begin{array}{c}
\xi_{0} \\
\omega_{0} \\
\zeta_{0} \\
R_{0}
\end{array}\right), \quad Z_{1}=\left(\begin{array}{c}
\xi_{1} \\
\omega_{1} \\
\zeta_{1} \\
R_{1}
\end{array}\right) \in \mathbb{R}^{9} \times S O(3)
$$


It is easy to check that there exist two $C^{2}$ functions $\widetilde{\zeta}:[0, T] \rightarrow \mathbb{R}^{3}, \widetilde{R}:[0, T] \rightarrow S O(3)$ such that

$$
\begin{gathered}
\widetilde{\xi}(0)=\xi_{0}, \widetilde{\omega}(0)=\omega_{0}, \widetilde{\zeta}(0)=\zeta_{0}, \widetilde{R}(0)=R_{0}, \\
\widetilde{\xi}(T)=\xi_{1}, \widetilde{\omega}(T)=\omega_{1}, \widetilde{\zeta}(T)=\zeta_{1}, \widetilde{R}(T)=R_{1} .
\end{gathered}
$$

If we set

$$
u(t)=B^{-1}(\dot{\tilde{z}}-A \widetilde{z}-E(\widetilde{z}))
$$

with $\widetilde{z}(t)=\left(\begin{array}{c}\widetilde{R}^{*} \dot{\widetilde{\zeta}} \\ \mathbb{S}^{-1}\left(\widetilde{R}^{*} \dot{\widetilde{R}}\right)\end{array}\right)$, then we clearly have $Z(0)=Z_{0}, Z(T)=Z_{1}$; thus the system is controllable in time $T$. We have shown that $\mathcal{Y}_{1}$ contains $\widetilde{\mathcal{Y}}_{1}$.

On the other hand, by comparing (3.8)-(3.9) and (4.6), we see that $B=\Lambda(\Psi)$. Since the mapping $\Lambda$ is continuous from $\mathcal{U}^{6}$ to $M_{6}(\mathbb{R})$ and since the set of invertible matrices is open in $M_{6}(\mathbb{R})$, we have that $\widetilde{\mathcal{Y}}_{1}$ is open in $\mathcal{U}^{6}$. We next check the density of $\widetilde{\mathcal{Y}}_{1}$. By Lemma 4.2, there exists $\widetilde{\Psi} \in \mathcal{U}^{6}$ such that $\Lambda(\widetilde{\Psi})=I_{6}$. For $\Psi \in \mathcal{U}^{6}$, we consider the sequence $\left(\Psi-\frac{1}{j} \widetilde{\Psi}\right)_{j \in \mathbb{N}^{*}}$, which converges to $\Psi$. It is easy to check that, excepting a finite number of values of $j$ the matrix $\Lambda\left(\Psi-\frac{1}{j} \widetilde{\Psi}\right)$ is invertible. We have thus shown that $\mathcal{Y}_{1}$ contains the set $\widetilde{\mathcal{Y}}_{1}$, which is open and dense in $\mathcal{U}^{6}$.

Proof of Theorem [1.2. As in the above proof, we first introduce the set $\widetilde{\mathcal{Y}}_{3}$ of those $\Psi$ for which $B$ given by (3.8) and (3.9) is invertible.

We can check that $\mathcal{Y}_{3}$ contains $\widetilde{\mathcal{Y}}_{3}$ as in the proof of Theorem 1.1] Moreover, using the fact that $B=\Lambda(\Psi)$, that the mapping $\Lambda$ is continuous from $\mathcal{W}^{6}$ to $M_{6}(\mathbb{R})$ and that the set of invertible matrices is open in $M_{6}(\mathbb{R})$, we get that $\widetilde{\mathcal{Y}}_{3}$ is open in $\mathcal{W}^{6}$. Finally, we verify the density of $\widetilde{\mathcal{Y}}_{3}$. By using again Lemma 4.2, we obtain the existence of $\widetilde{\Psi} \in \mathcal{W}^{6}$ such that $\Lambda(\widetilde{\Psi})=I_{6}$. Then, acting as in the above proof, we show that for all $\Psi \in \mathcal{W}^{6}$, there exists a sequence $\Psi_{n} \in \widetilde{\mathcal{Y}}_{3}$ such $\Psi_{n}$ converges toward $\Psi$.

5. Examples. In this section, we give some examples of families $\Psi$ of vector fields, defined on $\partial S$, for which the system (2.18)-(2.27) is controllable. Denote

- $\mathcal{F}_{0}=\left\{e_{1}, e_{2}, e_{3}, e_{1} \times y, e_{2} \times y, e_{3} \times y\right\}$;

- $\mathcal{F}_{1}=\left\{g^{(1)}, g^{(2)}, g^{(3)}, G^{(1)}, G^{(2)}, G^{(3)}\right\}$;

- $\mathcal{F}_{2}=\left\{\left(g^{(i)} \times n\right) \times n,\left(G^{(i)} \times n\right) \times n \mid i \in\{1,2,3\}\right\}$.

We remark that the families $\mathcal{F}_{1}$ and $\mathcal{F}_{2}$ have already been used in the previous sections.

The main result of this section is

Proposition 5.1. If $\Psi$ is one of the above families, then the system (2.18)-(2.27) is exactly controllable in any time $T>0$.

Proof. We first remark that it suffices to show that, for $\Psi \in\left\{\mathcal{F}_{0}, \mathcal{F}_{1}, \mathcal{F}_{2}\right\}$ the matrix $B$ defined by (3.8)-(3.10) is invertible.

If $\Psi=\mathcal{F}_{0}$, then $B=A$ so that $B$ is clearly invertible. 
If $\Psi=\mathcal{F}_{1}$, then

$$
B=\left(\begin{array}{cc}
m I_{3} & 0 \\
0 & J
\end{array}\right)^{-1}\left(\begin{array}{cc}
M & N \\
N^{*} & O
\end{array}\right)
$$

with

$$
M_{i, j}=-\int_{\partial S} g^{(i)} \cdot g^{(j)} \mathrm{d} \Gamma, N_{i, j}=-\int_{\partial S} g^{(i)} \cdot G^{(j)} \mathrm{d} \Gamma, O_{i, j}=-\int_{\partial S} G^{(i)} \cdot G^{(j)} \mathrm{d} \Gamma .
$$

According to Lemma 4.1, the system of vector functions $\left\{g^{(i)}, G^{(i)} \mid i \in\{1,2,3\}\right\}$ is linearly independent in $L^{2}\left(\partial S ; \mathbb{R}^{3}\right)$, so that the second matrix in the right-hand side of (5.1) is invertible. Consequently $B$ is invertible in the case $\Psi=\mathcal{F}_{1}$.

Assume then that $\Psi=\mathcal{F}_{2}$. Since, in this case, the vector fields belonging to $\Psi$ are tangential, formulas (3.8)-(3.10) yield

$$
B=\left(\begin{array}{cc}
m I_{3} & 0 \\
0 & J
\end{array}\right)^{-1}\left(\begin{array}{cc}
\widetilde{M} & \widetilde{N} \\
\widetilde{N}^{*} & \widetilde{O}
\end{array}\right),
$$

with

$$
\begin{aligned}
& \widetilde{M}_{i, j}=\int_{\partial S}\left[\left(g^{(i)} \times n\right) \times n\right] \cdot\left[\left(g^{(j)} \times n\right) \times n\right] \mathrm{d} \Gamma, \\
& \widetilde{N}_{i, j}=\int_{\partial S}\left[\left(g^{(i)} \times n\right) \times n\right] \cdot\left[\left(G^{(j)} \times n\right) \times n\right] \mathrm{d} \Gamma, \\
& \widetilde{O}_{i, j}=\int_{\partial S}\left[\left(G^{(i)} \times n\right) \times n\right] \cdot\left[\left(G^{(j)} \times n\right) \times n\right] \mathrm{d} \Gamma .
\end{aligned}
$$

By using again Lemma 2.1 from [8] the system of vector functions

$$
\left\{\left[\left(g^{(i)} \times n\right) \times n\right],\left[\left(G^{(i)} \times n\right) \times n\right] \mid i=1,3\right\}
$$

is linearly independent in $L^{2}\left(\partial S ; \mathbb{R}^{3}\right)$, so that the second matrix in the right-hand side of (5.2) is invertible. Consequently $B$ is invertible for $\Psi=\mathcal{F}_{2}$.

The fact that the above choice of the families $\mathcal{F}_{1}$ and $\mathcal{F}_{2}$ is physically relevant may be motivated by the following result:

Proposition 5.2. Assume that $\Psi=\left\{\psi_{i} \mid i=1, \ldots, k\right\}$ is a family of $C^{2}\left(\partial S ; \mathbb{R}^{3}\right)$. Then we have that

(1) if $\Psi \subset \mathcal{F}_{1}^{\perp}$ (the orthogonal is taken in $\left.L^{2}\left(\partial S ;\left(\mathbb{R}^{3}\right)\right)^{6}\right)$, then the system (3.28), (3.29) is not controllable;

(2) if $\Psi \subset \mathcal{V} \cap \mathcal{F}_{2}^{\perp}$, then the system (3.28), (3.29) is not controllable.

More precisely, in the above cases, the control $u$ does not act on the system (3.28), (3.29).

Proof. From (3.8)-(3.10) it follows that $B=0$; thus, in this case, the input function has no influence on the state of the system.

An interesting question is to know if the motion can be controlled by using less than six scalar inputs. This question is open in the general case. A partial answer is given by the two results below in the particular case where the rigid body is the unit ball.

We first show that by suppressing an appropriate element of one of the families $\mathcal{F}_{0}$, $\mathcal{F}_{1}$ and $\mathcal{F}_{2}$ (the families introduced at the beginning of this section) the resulting system is no longer controllable. 
Proposition 5.3. Assume that $S$ is the unit ball in $\mathbb{R}^{3}$. Then, for every $i \in\{0,1,2\}$ there exists a set containing five elements $\Psi_{i} \subset \mathcal{F}_{i}$ such that the system (3.28), (3.29) is not controllable.

Proof. With the assumption that $S$ is the unit ball in $\mathbb{R}^{3}$ the fields $g^{(i)}, G^{(i)}$ are explicitly given (see, for instance, [10, pp. 163, 169]) by the formulas

$$
g^{(i)}(y)=\frac{3}{2} e_{i}, \quad G^{(i)}(y)=3 e_{i} \times y, \quad i \in\{1,2,3\} .
$$

In the next calculation we use the quantities $\epsilon_{i j k}$ which are the components of the classical permutation tensor, i.e., the quantities $\epsilon_{i j k}$ are skew-symmetric with respect to any couple of indexes and $\epsilon_{123}=1$.

We next inject the expressions (5.3) into the formulas (3.5) and (3.6) which define the matrices $K, C, S$ and $\Omega$. We obtain

$$
\begin{aligned}
K_{i, j}=-\frac{3}{2} \int_{\partial S} \delta_{i j} \mathrm{~d} \Gamma=-6 \pi \delta_{i j} \quad \forall i, j \in\{1,2,3\} \\
C_{i, j}=-\frac{3}{2} \int_{\partial S} \epsilon_{j k l} y_{k} \delta_{i l} \mathrm{~d} \Gamma=0 \quad \forall i, j \in\{1,2,3\} \\
\Omega_{i, j}=-3\left[\int_{\partial S} y \times\left(e_{i} \times y\right)\right]_{j} \\
=-3 \int_{\partial \Omega} \epsilon_{j p q} y_{p} \epsilon_{q r s} \delta_{i r} y_{s} \\
=-4 \pi \epsilon_{j p q} \epsilon_{q r s} \delta_{i r} \delta_{p s} \\
=-4 \pi \epsilon_{j p q} \epsilon_{r p q} \delta_{i r} \\
=-8 \pi \delta_{j r} \delta_{i r} \\
=-8 \pi \delta_{i j} .
\end{aligned}
$$

Consequently we have

$$
K=-6 \pi I_{3}, \quad C=0, \quad \Omega=-8 \pi I_{3} .
$$

The above relations, combined with (3.7), imply that

$$
A=\left(\begin{array}{cc}
-\frac{3}{2 \rho} I_{3} & 0 \\
0 & -\frac{15}{\rho} I_{3}
\end{array}\right)
$$

We next take $\Psi=\left\{e_{1}, e_{2}, e_{3}, e_{1} \times y, e_{2} \times y\right\}$, which is a five-element subset of $\mathcal{F}_{0}$. With the above choice of $\Psi$ and by using (3.8)-(3.10) we obtain

$$
B=\left(\begin{array}{ccccc}
-\frac{3}{2 \rho} & 0 & 0 & 0 & 0 \\
0 & -\frac{3}{2 \rho} & 0 & 0 & 0 \\
0 & 0 & -\frac{3}{2 \rho} & 0 & 0 \\
0 & 0 & 0 & -\frac{15}{\rho} & 0 \\
0 & 0 & 0 & 0 & -\frac{15}{\rho} \\
0 & 0 & 0 & 0 & 0
\end{array}\right) .
$$


Moreover, since $S$ is a ball its inertia matrix $J$ is a scalar matrix so that the function $E$ defined by (3.15) reduces to

$$
E\left(\left(\begin{array}{l}
a \\
b
\end{array}\right)\right)=\left(\begin{array}{c}
b \times a \\
0
\end{array}\right), \quad a, b \in \mathbb{R}^{3} .
$$

By using the above form of $A, B$ and $E$ we see that the equation for $\omega_{3}$ in (3.28), (3.29) reduces to

$$
\dot{\omega}_{3}=-\frac{15}{\rho} \omega_{3}
$$

which is independent of the control $u$. This clearly implies that the full system (3.28), (3.29) is not controllable.

The cases in which the family $\Psi$ is a subset of $\mathcal{F}_{1}$ or of $\mathcal{F}_{2}$ can be tackled in a similar way, so we skip the corresponding calculations.

The next result states that if we consider only the system (3.28) not involving the position vector $\zeta$ and the rotation $R$ and we assume that $S$ is the unit ball of $\mathbb{R}^{3}$, we can locally control the velocity field with only three scalar inputs. More precisely, the following result holds.

Proposition 5.4. There exists an open and dense subset $\mathcal{Y}_{4}$ of $\mathcal{W}^{3}$ such that the system (3.28) is locally controllable in a neighborhood of the origin for any family $\left\{\psi_{1}, \psi_{2}, \psi_{3}\right\}$ $\subset \mathcal{Y}_{4}$.

Proof. It is well known that the local controllability follows from the controllability of the linearized system which, in our case, reduces to

$$
\dot{z}(t)=A z(t)+B u(t),
$$

with the matrix $A$ given by (5.4) and the matrix $B \in M_{6 \times 3}(\mathbb{R})$ given by (3.10). The eigenvectors of $A$ have the form

$$
\left(\begin{array}{l}
a \\
0
\end{array}\right) \text { or }\left(\begin{array}{l}
0 \\
a
\end{array}\right), \quad a \in \mathbb{R}^{3} \backslash\{0\} .
$$

By applying the Hautus test (see, for instance, Sontag [18, p. 94]) we obtain that (3.28) is controllable if and only if the matrices $B^{(1)}$ and $B^{(2)}$ in (3.10) are invertible. In order to finish the proof it suffices to show that the set of families $\left\{\psi_{1}, \psi_{2}, \psi_{3}\right\} \in \mathcal{W}^{3}$ such that the $3 \times 3$ matrices $B^{(1)}$ and $B^{(2)}$ defined by (3.8) and (3.9) are invertible is open and dense. In order to do that, consider the linear operator $\Lambda_{4}: \mathbb{W}^{\prime} \rightarrow\left[M_{3}(\mathbb{R})\right]^{2}$,

$$
\Lambda\left(\begin{array}{c}
\psi_{1} \\
\psi_{2} \\
\psi_{3}
\end{array}\right)=\left(\begin{array}{l}
\left(\int_{\partial S} g^{(i)} \cdot \psi_{j} \mathrm{~d} \Gamma\right)_{i, j \in\{1,2,3\}} \\
\left(\int_{\partial S} G^{(i)} \cdot \psi_{j} \mathrm{~d} \Gamma\right)_{i, j \in\{1,2,3\}}
\end{array}\right),
$$

where

$$
\mathbb{W}^{\prime}=\left\{\left(\varphi_{i}\right) \in L^{2}\left(\partial S ; \mathbb{R}^{3}\right)^{3} \mid \varphi_{i}=0 \text { outside } \Gamma, \quad \varphi_{i} \cdot n=0 \text { on } \partial S, i \in\{1,2,3\}\right\} .
$$


A simple calculation shows that for all $(C, D) \in M_{3}(\mathbb{R})$,

$$
\Lambda_{4}^{*}\left(\begin{array}{l}
C \\
D
\end{array}\right)=\left(\begin{array}{c}
\sum_{k=1}^{3}\left(C_{k, 1}\left(g^{(k)} \times n\right) \times n+D_{k, 1}\left(G^{(k)} \times n\right) \times n\right) \\
\sum_{k=1}^{3}\left(C_{k, 2}\left(g^{(k)} \times n\right) \times n+D_{k, 2}\left(G^{(k)} \times n\right) \times n\right) \\
\sum_{k=1}^{3}\left(C_{k, 3}\left(g^{(k)} \times n\right) \times n+D_{k, 3}\left(G^{(k)} \times n\right) \times n\right)
\end{array}\right) \quad \text { on } \Gamma .
$$

Using Lemma 4.1 (2), we deduce that $\Lambda_{4}^{*}$ is one to one. The end of the proof is completely similar to the proof of Theorem 1.1 .

6. Concluding remarks. The results presented here illustrate the controllability properties of a dynamical system modelling the motion of some ocean micro-organisms. Within this model it would be interesting to study in a detailed way the influence of the form of the micro-organism on the controllability properties of the system. We have seen that for a micro-organism having the form of the ball at least six scalar controls seem to be necessary. We conjecture that for less symmetric forms controllability of the full system could be obtained with less than six scalar inputs. One of the major simplifying assumptions in the present work is that we neglect the term containing the partial derivative with respect to the time in the equations of the fluid. Further development is therefore required to include this term in the analysis. This question seems difficult since the introduction of this term makes the associated dynamical system genuinely infinitedimensional. An investigation using this more comprehensive model would provide more insight into the swimming mechanism of micro-organisms.

Acknowledgements. The three authors have been supported by the ANCIF associated research team of INRIA. The first author was partially supported by Conicyt under grant Fondecyt 10150332 and by the Center for Mathematical Modelling from Chile.

\section{REFERENCES}

[1] J. R. Blake and S. R. Otto, Ciliary propulsion, chaotic filtration and a "blinking" Stokeslet, J. Engrg. Math. 30 (1996), no. 1-2, 151-168, The centenary of a paper on slow viscous flow by the physicist H. A. Lorentz. MR.1396365 (97e:76098)

[2] J. R. Blake, A finite model for ciliated micro-organisms, J. Biomech. 6 (1973), 133-140.

[3] Christopher Brennen, An oscillating-boundary-layer theory for ciliary propulsion, J. Fluid Mech. 65 (1974), 799-824.

[4] Christopher Brennen and H. Winet, Fluid mechanics of propulsion by cilia and flagella, Ann. Rev. Fluid Mech. 9 (1977), 339-398.

[5] Stephen Childress, Mechanics of swimming and flying, Cambridge Studies in Mathematical Biology, vol. 2, Cambridge University Press, Cambridge, 1981. MR665099|(84a:76050)

[6] Caroline Fabre and Gilles Lebeau, Prolongement unique des solutions de l'equation de Stokes, Comm. Partial Differential Equations 21 (1996), no. 3-4, 573-596. MR1387461 (97c:35156)

[7] Giovanni P. Galdi, An introduction to the mathematical theory of the Navier-Stokes equations. Vol. I, Springer Tracts in Natural Philosophy, vol. 38, Springer-Verlag, New York, 1994, Linearized steady problems. MR1284205 (95i:35216a)

[8] _ On the steady self-propelled motion of a body in a viscous incompressible fluid, Arch. Rational Mech. Anal. 148 (1999), no. 1, 53-88. MR1715453(2000h:76186) 
[9] - On the motion of a rigid body in a viscous liquid: A mathematical analysis with applications, Handbook of mathematical fluid dynamics. Vol. I, North-Holland, Amsterdam, 2002, pp. 653-791. MR1942470 (2003j:76024)

[10] John Happel and Howard Brenner, Low Reynolds number hydrodynamics with special applications to particulate media, Prentice-Hall Inc., Englewood Cliffs, N.J., 1965. MR0195360 (33:3562)

[11] S. R. Keller and T. Y. Wu, A porous prolate-spheroidal model for ciliated micro-organisms, J. Fluid Mech. 80 (1977), 259-278.

[12] Gen Komatsu, Analyticity up to the boundary of solutions of nonlinear parabolic equations, Comm. Pure Appl. Math. 32 (1979), no. 5, 669-720. MR533297 (82j:35084)

[13] James Lighthill, Mathematical biofluiddynamics, Society for Industrial and Applied Mathematics, Philadelphia, 1975. MR0469320 (57:9113)

[14] Charles B. Morrey, Jr., Multiple integrals in the calculus of variations, Die Grundlehren der mathematischen Wissenschaften, Band 130, Springer-Verlag New York, Inc., New York, 1966. MR0202511 $(34: 2380)$

[15] Jorge Alonso San Martín, Victor Starovoitov, and Marius Tucsnak, Global weak solutions for the two-dimensional motion of several rigid bodies in an incompressible viscous fluid, Arch. Rational Mech. Anal. 161 (2002), no. 2, 113-147. MR1870954 (2002j:35259)

[16] Denis Serre, Chute libre d'un solide dans un fluide visqueux incompressible. Existence, Japan J. Appl. Math. 4 (1987), no. 1, 99-110. MR899206 (89m:76032)

[17] Ana Leonor Silvestre, On the slow motion of a self-propelled rigid body in a viscous incompressible fluid, J. Math. Anal. Appl. 274 (2002), no. 1, 203-227. MR1936694 (2003j:76030)

[18] Eduardo D. Sontag, Mathematical control theory, second ed., Texts in Applied Mathematics, vol. 6, Springer-Verlag, New York, 1998, Deterministic finite-dimensional systems. MR1640001 (99k:93001)

[19] Takéo Takahashi and Marius Tucsnak, Global strong solutions for the two-dimensional motion of an infinite cylinder in a viscous fluid, J. Math. Fluid Mech. 6 (2004), no. 1, 53-77. MR2027754 (2004k:76035)

[20] Geoffrey Taylor, Analysis of the swimming of microscopic organisms, Proc. Roy. Soc. London Ser. A. 209 (1951), 447-461. MR0045521(13:596a) 